\title{
EDITORIAL
}

\section{Can osteoarthritis be treated with light?}

\author{
Michael R Hamblin ${ }^{1,2,3}$
}

See related research by Alves et al. http://arthritis-research.com/content/15/5/R116

\begin{abstract}
Osteoarthritis is becoming more problematic as the population ages. Recent reports suggest that the benefit of anti-inflammatory drugs is unimpressive and the incidence of side effects is worrying. Low-level laser (light) therapy (LLLT) is an alternative approach with no known side effects and with reports of substantial therapeutic efficacy in osteoarthritis. In this issue of Arthritis Research \& Therapy, Alves and colleagues used a rat model of osteoarthritis produced by intra-articular injection of the cartilage-degrading enzyme papain to test 810-nm LLLT. A single application of LLLT produced significant reductions in inflammatory cell infiltration and inflammatory cytokines 24 hours later. A lower laser power was more effective than a higher laser power. However, more work is necessary before the title question can be answered in the affirmative.
\end{abstract}

In this issue of Arthritis Research \& Therapy, Alves and colleagues [1] reported an interesting preclinical study suggesting that near-infrared light applied to the rat knee has significant benefits on joint inflammation in an animal model of osteoarthritis. One in every two persons in the US will experience some form of osteoarthritis in their lifetime, and the incidence is $80 \%$ in those over 75 [2]. Treatment is with analgesics (acetaminophen), nonsteroidal anti-inflammatory drugs (NSAIDs) (ibuprofen and diclofenac), and COX2 inhibitors (Celebrex, Pfizer Inc., New York, NY, USA), but these therapies have significant risks of adverse effects, such as gastrointestinal bleeding with NSAIDs and myocardial infarction with COX2 inhibitors [3]. Low-level laser (light) therapy (LLLT) is a rapidly growing alternative approach to many medical conditions that require relief from pain

\footnotetext{
Correspondence: hamblin@helix.mgh.harvard.edu

${ }^{1}$ Wellman Center for Photomedicine, Massachusetts General Hospital,

BAR414, 40 Blossom Street, Boston, MA 02114, USA

Department of Dermatology, Harvard Medical School, 25 Shattuck Street,

Boston, MA 02115, USA

Full list of author information is available at the end of the article
}

and inflammation, stimulation of healing, and prevention of tissue death after injury or infarction [4]. The red or near-infrared photons are absorbed in cytochrome $c$ oxidase (unit IV of the mitochondrial respiratory chain), thereby increasing mitochondrial respiration and ATP production and initiating signaling pathways mediated by reactive oxygen species, nitric oxide, and cyclic AMP, ultimately leading to activation of several transcription factors [5]. A large number of studies have examined the anti-inflammatory effects of LLLT and have reported reductions in inflammatory cell (neutrophils, macrophages, lymphocytes, and mast cells) infiltration in multiple pathologies and have shown reductions in several inflammatory cytokines, such as ILs and TNF- $\alpha$. Many observers have reported that there is a biphasic dose response that operates in LLLT, and this means that increasing the total energy or the rate of energy delivery (power density) may be counterproductive, giving less overall benefit compared with lower doses of LLLT [6]. LLLT has been used clinically in osteoarthritis for many years but is still considered controversial. Although a Cochrane review [7] reported mixed and conflicting results, a subsequent analysis conducted by Bjordal and colleagues concluded that the Cochrane review conclusion was neither robust nor valid. Further sensitivity analyses with inclusion of valid non-included trials, performance of missing follow-up, and subgroup analyses revealed consistent and highly significant results in favor of active LLLT for osteoarthritis' [8]. This disagreement in the literature suggests that much more work comprising animal studies, clinical trials, and systematic reviews will need to be done before LLLT becomes accepted as a valid therapy.

Alves and colleagues [1] used a clinically relevant model of osteoarthritis in which the proteolytic enzyme papain is injected into the rat knee joint, where it degrades the cartilage, thus causing inflammation. The knees received a single application of $4 \mathrm{~J}$ of energy from an 808-nm laser to the medial and lateral aspects of the knee, delivered at either 50 or $100 \mathrm{~mW}$, and the rats were sacrificed after 24 hours. The authors found a 
significant reduction in inflammatory cells in fluid from synovial washing with both power levels but a bigger reduction in macrophages at the $50-\mathrm{mW}$ power level. Reductions in IL- $1 \beta$ and IL- 6 mRNA were found, with $50 \mathrm{~mW}$ being better than $100 \mathrm{~mW}$, whereas a bigger reduction in TNF- $\alpha$ was seen with $100 \mathrm{~mW}$. The authors hypothesized that the twice-longer illumination time needed to deliver the same energy at half the power may have been responsible for the better effect of the 50-mW laser application, and this agrees with a previous study that examined zymosan-induced arthritis in the rat knee [9] and found that a longer illumination time was more effective. Previous work from this group [10] had shown that LLLT in the same model increased angiogenesis and the amount of squamous epithelium while decreasing fibrosis in the joint. These results provide additional justification for the use of LLLT (especially near-infrared laser that has the tissue penetration required for joints) as a treatment for osteoarthritis. Nevertheless, it should be pointed out that the study was only an animal model, not a clinical study in human disease, and further studies will be necessary to define the benefits of LLLT in osteoarthritis. In addition to the demonstrated anti-inflammatory effects, LLLT may have benefits provided by its ability to act on nerves by reducing pain transmission and activating endogenous opioid receptors [11].

\footnotetext{
Abbreviations

IL: Interleukin; LLLT: Low-level laser (light) therapy; NSAID: Non-steroidal antiinflammatory drug; TNF-a: Tumor necrosis factor-alpha.
}

\section{Competing interests}

The author declares that he has no competing interests.

\section{Acknowledgments}

Research in the author's laboratory is supported by National Institutes of Health grant R01A1050875.

\section{Author details}

'Wellman Center for Photomedicine, Massachusetts General Hospital, BAR414, 40 Blossom Street, Boston, MA 02114, USA. 'Department of Dermatology, Harvard Medical School, 25 Shattuck Street, Boston, MA 02115, USA. ${ }^{3}$ Harvard-MIT Division of Health Sciences and Technology, 77 Massachusetts Avenue, Cambridge, MA 02139, USA.

Published: 29 Oct 2013

\section{References}

1. Alves AC, Vieira RP, Leal-Junior EC, Dos Santos SA, Ligeiro AP, Albertini R, Junior JA, de Carvalho PD: Effect of low level laser therapy on the expression of inflammatory mediators and on neutrophils and macrophages in acute joint inflammation. Arthritis Res Treat 2013, 15:R116.

2. Arden N, Nevitt MC: Osteoarthritis: epidemiology. Best Pract Res Clin Rheumatol 2006, 20:3-25.

3. Cheng DS, Visco CJ: Pharmaceutical therapy for osteoarthritis. PM R 2012, 4:S82-S88.

4. Chung H, Dai T, Sharma SK, Huang YY, Carroll JD, Hamblin MR: The nuts and bolts of low-level laser (light) therapy. Ann Biomed Eng 2012, 40:516-533.

5. Chen AC, Arany PR, Huang YY, Tomkinson EM, Sharma SK, Kharkwal GB, Saleem T, Mooney D, Yull FE, Blackwell TS, Hamblin MR: Low-level laser therapy activates NF-kB via generation of reactive oxygen species in mouse embryonic fibroblasts. PLOS ONE 2011, 6:e22453.

6. Huang YY, Chen AC, Carroll JD, Hamblin MR: Biphasic dose response in low level light therapy. Dose Response 2009, 7:358-383.

7. Brosseau L, Welch V, Wells G, DeBie R, Gam A, Harman K, Morin M, Shea B, Tugwell P: Low level laser therapy (Classes I, II and III) for treating osteoarthritis. Cochrane Database Syst Rev 2004, 3, CD002046.

8. Bjordal JM, Bogen B, Lopes-Martins RA, Klovning A: Can Cochrane Reviews in controversial areas be biased? A sensitivity analysis based on the protocol of a Systematic Cochrane Review on low-level laser therapy in osteoarthritis. Photomed Laser Surg 2005, 23:453-458.

9. Castano AP, Dai T, Yaroslavsky I, Cohen R, Apruzzese WA, Smotrich MH, Hamblin MR: Low-level laser therapy for zymosan-induced arthritis in rats: importance of illumination time. Lasers Surg Med 2007, 39:543-550.

10. da Rosa AS, dos Santos AF, da Silva MM, Facco GG, Perreira DM, Alves AC, Leal Junior EC, de Carvalho Pde T: Effects of low-level laser therapy at wavelengths of 660 and $808 \mathrm{~nm}$ in experimental model of osteoarthritis. Photochem Photobiol 2012, 88:161-166.

11. Cidral-Filho FJ, Mazzardo-Martins L, Martins DF, Santos AR: Light-emitting diode therapy induces analgesia in a mouse model of postoperative pain through activation of peripheral opioid receptors and the Larginine/nitric oxide pathway. Lasers Med Sci 2013. Epub ahead of print.

\subsection{6/ar4354}

Cite this article as: Hamblin: Can osteoarthritis be treated with light? Arthritis Research \& Therapy 2013, 15:120 